\title{
Perspectives
}

uclear magnetic resonance (NMR) ${ }^{1}$ spectroscopy, which has been used by chemists and physicists for more than 30 years, is now becoming a powerful technique in the medical sciences because of its unique ability to monitor intracellular metabolites noninvasively. The fundamental principle underlying NMR spectroscopy is that nuclei of certain atoms, such as hydrogen $\left({ }^{1} \mathrm{H}\right)$, phosphorus $\left({ }^{31} \mathrm{P}\right)$, carbon $\left({ }^{13} \mathrm{C}\right)$, fluorine $\left({ }^{19} \mathrm{~F}\right)$, and sodium $\left({ }^{23} \mathrm{Na}\right)$, are magnetic dipoles. In the presence of a strong magnetic field, the nuclei of these atoms align with or against the field in much the same way that a bar magnet tends to align itself with the earth's magnetic field. A pulse of perturbing electromagnetic energy at the resonant frequency is then used to tip the nuclei away from their orientation along the magnetic field lines. When the perturbing radiofrequency pulse is stopped, the nuclei return to their original lower energy orientations; in the process, they emit energy which can be picked up by a radiofrequency receiver and transformed, with the aid of a computer, into a spectrum. The different nuclei resonate in the same magnetic field at very different frequencies. For example, at a field of $8.4 \mathrm{~T}$, the ' $\mathrm{H}$-resonance is at $360 \mathrm{MHz}$ while ${ }^{13} \mathrm{C}$ and ${ }^{31} \mathrm{P}$ are near 90.5 and 145.8 $\mathrm{MHz}$, respectively. Members of the same nuclear species occupying different positions in a molecule resonate at slightly different frequencies. The individual peaks of the spectrum arise from differences in the chemical bondings of individual

Received for publication 17 May 1984 and in revised form 12 June 1984.

1. Abbreviations used in this paper: NMR, nuclear magnetic resonance; $\mathrm{P}_{\mathrm{i}}$, inorganic phosphate; $\mathrm{PCr}$, phosphocreatine.

J. Clin. Invest.

(C) The American Society for Clinical Investigation, Inc.

$0021-9738 / 84 / 10 / 1127 / 05 \quad \$ 1.00$

Volume 74, October 1984, 1127-1131

\section{Nuclear Magnetic Resonance Spectroscopy in Diagnostic and Investigative Medicine}

\author{
Gerald I. Shulman, Jeffry R. Alger, James W. Prichard, \\ and Robert G. Shulman \\ Department of Molecular Biophysics and Biochemistry, Yale \\ University, New Haven, Connecticut 06511; Diabetes Unit, \\ Massachusetts General Hospital, Boston, Massachusetts 02114; \\ Department of Diagnostic Radiology, Yale University, New \\ Haven, Connecticut 06511; Department of Neurology, Yale \\ University School of Medicine, New Haven, Connecticut 06511
}

nuclei. The areas of the resonance peaks are proportional to the concentrations of the individual nuclei resonating at that particular frequency. The same basic principles of NMR are used in imaging experiments, which, however, must be distinguished from NMR spectroscopy. In imaging, the strong signal from protons, mostly those of tissue water, is detected in a spatially encoded way which allows construction of a two- or three-dimensional picture showing structure, mainly from the variation in water density. In spectroscopy, weaker signals from nonwater protons and other nuclei are used to obtain specific chemical information from tissue.

NMR spectroscopy requires powerful magnets capable of generating magnetic fields several thousand times that of the earth. During the past several years, technological advances have permitted construction of magnets large enough to accommodate humans. Consequently, the study of human metabolism by NMR techniques is becoming a reality. Conventional spectroscopic techniques are not suitable for most studies of whole animals because of the need to localize the signal source to a specific region in the animal. Presently, the best solution to this problem is resolved by surface coils, which are simply loops of wire placed on the surface that detect signals from an approximately disk-shaped region of tissue below the coil. This method, which was developed by Ackerman et al. (1), is adequate for some kinds of metabolic studies on large organs. Other localization techniques based on NMR imaging methods are being developed and show considerable promise (2-8).

\section{NMR spectroscopy as an investigative tool}

NMR spectroscopy has several advantages over other biochemical techniques. It is nondestructive, noninvasive, and is thought to be free of biologic hazard (9). The time course of a metabolic reaction in a single subject can be followed with spectra accumulated every few minutes, in contrast to the traditional 
method of freeze clamping, in which each time point represents a separate preparation. An example of this can be seen in Fig. 1 in which ${ }^{31} \mathrm{P}$-spectra of a rabbit brain before, during, and after hypoxia were obtained by using a surface coil placed on the animal's scalp. The top spectrum was performed under normoxic conditions. When hypoxia was induced (middle spectrum), there was a decrease in phosphocreatine ( $\mathrm{PCr}$ ) stores accompanied by an increase in inorganic phosphate $\left(P_{i}\right)$, a slowing of the electroencephalogram, and a slight fall in ATP levels. When normoxic conditions were restored (bottom spectrum), $\mathrm{PCr}$ and $\mathrm{P}_{\mathrm{i}}$ levels reverted to normal as did the electroencephalogram. Intracellular $\mathrm{pH}$ was also determined by monitoring the chemical shift of the $P_{i}$ peak (10) and was found to decrease reversibly during hypoxia. Furthermore, NMR provides information about the intracellular environment of metabolites which may be lost or distorted during the process of freeze clamping. For instance, freeze clamping techniques appear to overestimate intracellular concentrations of free ADP when compared with NMR methods. This is thought to be due to the rapid hydrolysis of high energy phosphate during freeze clamping and extraction, or to the fact that most of the ADP in intact tissue is tightly bound to the proteins of myofilaments (11). Only mobile compounds generate NMR signals, since immobilization gives rise to signals that are too broad to be observed.

NMR can also be used to measure the fluxes of certain enzyme reactions in vivo. The method used is magnetization transfer, a type of magnetic labeling experiment in which the magnetization of one chemical species is perturbed and the perturbation transferred to a second species if the two are in rapid exchange with each other. The rate of interconversion can be obtained from the extent to which the second species is affected. The kinetics of creatine kinase and ATP synthetase have been studied in living animals in this way (12).

Another capability of NMR involves enriching isotopes of low natural abundance such as ${ }^{13} \mathrm{C}$. NMR studies using ${ }^{13} \mathrm{C}$ enrichment can detect the distribution of labeled carbons within a molecule and can measure the amount of label at each site. This type of information is similar to that obtained by use of ${ }^{14} \mathrm{C}$-isotopes, but with the advantage that the metabolic intermediates need not be isolated and purified to determine the amount of labeled compounds, or chemically degraded to determine the label distribution within the molecule. In appropriate systems, the metabolism of individual carbon atoms can be followed through enzymatic steps. Such methods have been used successfully to study glycolysis in suspensions of Escherichia coli (13) and yeast (14), and in the heart (15) and brain (16) in vivo. The gluconeogenic flux has been followed from ${ }^{13} \mathrm{C}$-labeled alanine and glycerol in hepatocytes $(17,18)$ and in perfused livers (19). In double label experiments, the ${ }^{13} \mathrm{C}$ results have been found to be in close agreement with measurements on the same system using labeled ${ }^{14} \mathrm{C}$-isotopes (20). In vivo labeling of glycogen in rat liver (21) and of amino

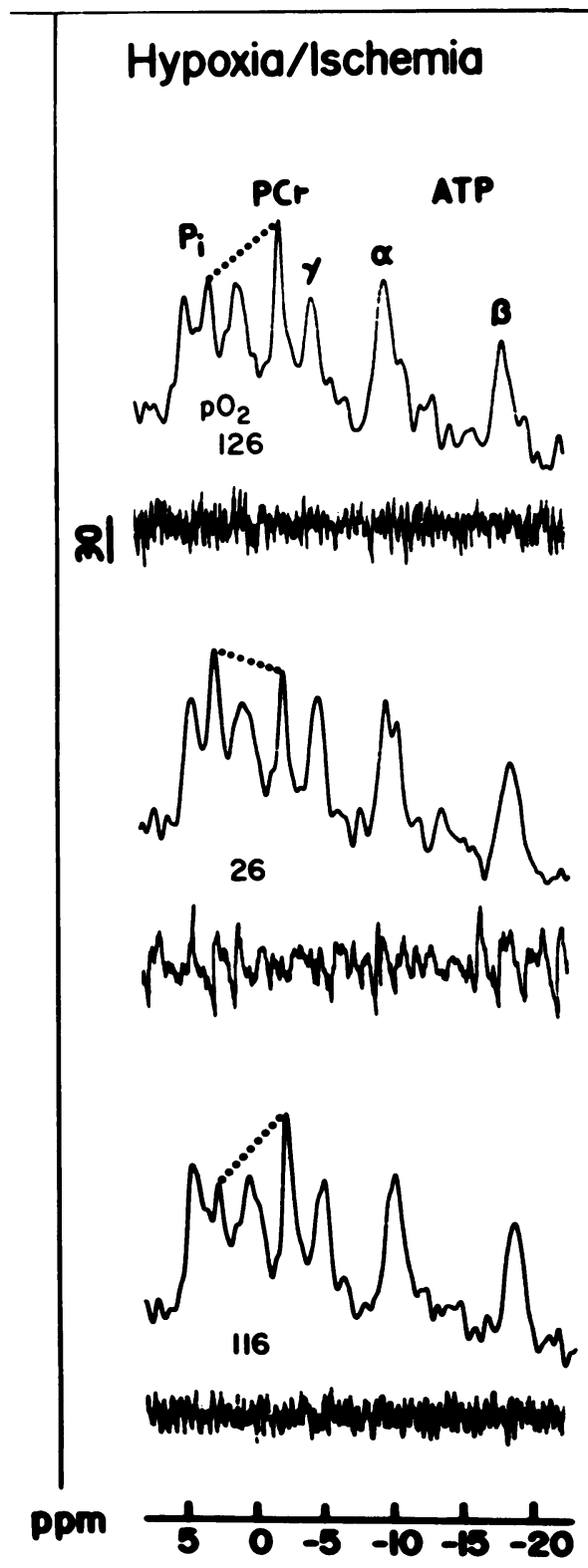

Figure 1. ${ }^{31} \mathrm{P}-\mathrm{NMR}$ spectra and electroencephalograms of a rabbit brain before (top), during (middle), and after (bottom) hypoxia, obtained at $32 \mathrm{MHz}$ using a surface coil placed against the animal's scalp. The $P_{i}$ and $P C r$ resonances which are joined by dotted lines in all spectra, and those of $\gamma, \alpha$, and $\beta$ phosphate residues of ATP are indicated on the upper trace. Unlabeled peaks to the left and right of the $P_{i}$ peak are currently attributed to phosphomonoester and phosphodiester residues, respectively. Arterial oxygen tension is given beneath the spectra (torr). All EEG traces are $20 \mathrm{~s}$ long and were recorded during accumulation of the spectra above them; the vertical calibration bar is in microvolts. 
acids and lactate in rabbit brain (16) has been demonstrated. There is no theoretical barrier to the use of similar methods in humans, but that has not yet been attempted.

Development of ${ }^{1} \mathrm{H}$-NMR for the study of metabolism in vivo is quite recent compared with the ${ }^{31} \mathrm{P}$ and ${ }^{13} \mathrm{C}$ methods. Although the relative sensitivity of the ${ }^{1} \mathrm{H}$ nucleus is greater than ${ }^{31} \mathrm{P}$ and ${ }^{13} \mathrm{C}$, the high concentration of tissue water relative to metabolites of interest and the small frequency range of the ${ }^{1} \mathrm{H}$-spectrum (10 parts per million [ppm]) compared with the spectrum of phosphate compounds $(40 \mathrm{ppm})$ or ${ }^{13} \mathrm{C}(200 \mathrm{ppm})$ have limited its use. The first in vivo high resolution 'H-NMR spectrum of human dystrophic muscle appeared in 1982 (22), but only resonances attributable to the protons of the fatty acid chains of acyl glycerides could be distinguished, and no small metabolites were detected due to the low resolution and the presence of large water and fatty acid resonances. More recently, Behar et al. (23), using a surface coil placed over a rat skull and newly developed methods for suppressing the water proton signal, were able to detect cerebral lactate, aspartate, glutamate, and gamma-aminobutyric acid levels in the rat brain in vivo and to monitor changes in them caused by hypoxia $(23,24)$ and hypoglycemia $(16)$. This technique shows considerable promise for in vivo studies in man because of the inherently greater sensitivity of the proton compared with other detectable nuclei.

\section{NMR as a diagnostic tool}

The diagnostic value of NMR has already been demonstrated in certain instances of inherited disorders of muscle metabolism $(11,26,27)$. However, it should be emphasized that NMR spectroscopy is still under development for this purpose and will not in the near future replace conventional biopsies and chemical measurements of body fluids. All clinical NMR spectroscopic studies to date have measured high energy phosphate compounds, $\mathrm{P}_{i}$ 's, and intracellular $\mathrm{pH}$ in the ${ }^{31} \mathrm{P}$-spectrum. The first clinical study was performed by Ross et al. (26) on a patient with McArdle's disease (muscle phosphorylase $a$ deficiency). The condition was demonstrated by observation of the failure of ischemic exercise to lower intracellular $\mathrm{pH}$ in forearm muscles of the patient, in contrast to the marked acidosis observed under these conditions in normal subjects. By using similar methods, these workers also demonstrated abnormal recovery of $\mathrm{PCr}$ and $\mathrm{pH}$ after exercise in two sisters with NADH coenzyme $Q$ reductase deficiency (27). Edwards et al. (11) demonstrated the usefulness of muscle NMR in diagnosing phosphofructokinase deficiency, which manifested itself by almost constant intracellular $\mathrm{pH}$ together with production of large amounts of sugar phosphates in muscle during aerobic and anaerobic exercise. ${ }^{31} \mathrm{P}-\mathrm{NMR}$ has also been used to study patients with Duchenne muscular dystrophy. In these patients, the intracellular $\mathrm{pH}$ of resting muscle was significantly more alkaline than in normal subjects and the ratio of $\mathrm{PCr}$ to ATP was low (22).
Cady et al. obtained ${ }^{31} \mathrm{P}-\mathrm{NMR}$ spectra from the brains of several infants who had suffered severe intrapartum asphyxia or had congenital anomalies (28). They measured the ratio of $\mathrm{PCr}$ to $\mathrm{P}_{i}$ and found that the ratio increased with clinical improvement. In two infants, grossly abnormal ${ }^{31} \mathrm{P}$-spectra antedated the detection by ultrasound of large porencephalic cysts. Similar techniques were used to monitor the response to chemotherapy of an alveolar rhabdomyosarcoma on the back of a hand of a young woman (29).

${ }^{13} \mathrm{C}$ - and ${ }^{1} \mathrm{H}-\mathrm{NMR}$ have not yet been used in clinical diagnosis. However, a few reports have demonstrated their potential usefulness by distinguishing normal from dystrophic muscle (22) and detecting certain disorders of hepatic glycogen metabolism in the rat (30).

\section{Limitations of NMR spectroscopy in vivo}

The major limitations are sensitivity and spectral resolution. Sensitivity of in vivo NMR for ${ }^{13} \mathrm{C}$ - and ${ }^{31} \mathrm{P}$-signals is about the same and requires concentrations of $\sim 1 \mathrm{mM}$ to obtain reasonable signal-to-noise within several minutes of accumulation. This is obtained in the ${ }^{31} \mathrm{P}$-spectra for the naturally occurring ATP, PCr, $P_{i}$, and some other highly concentrated metabolites. For ${ }^{13} \mathrm{C}$, it is necessary to enrich metabolites by introducing labeled precursors to reach millimolar levels of ${ }^{13} \mathrm{C}$-labeled compounds. However, in highly concentrated storage compounds such as glycogen (31) and certain lipids (32), it has been possible to detect ${ }^{13} \mathrm{C}$-peaks in their $1.1 \%$ natural abundance because the glucose and fatty acid moieties are present in relatively high concentrations.

The problem of resolution concerns distinguishing signals from members of the same nuclear species whose resonant frequencies are close together because their chemical, and hence magnetic, environments are similar. This problem is greatly reduced by new methods of observation which employ a technique called decoupling to simplify spectra by manipulating magnetic interactions among neighboring nuclei $(33,34)$.

\section{Safety}

While it is generally believed that whole body NMR is safe, there are several potential hazards that need to be assessed carefully before NMR studies on humans become routine (9). These derive from: $(a)$ the static magnetic field, $(b)$ the induction of electrical currents in the body, and (c) decoupling with a radiofrequency field.

Apart from the obvious danger to patients with cardiac pacemakers and the possibility that implanted ferromagnetic objects in the body could be caused to move by a strong magnetic field, there is currently no verified evidence for any detrimental effect of a static magnetic field. Years of experience with particle accelerator magnets of similar field strength have provided much data on the matter (35).

While electrical currents can be induced in the body by 
sudden quenching of a magnetic field or by rapid movements within a static magnetic field, present data show that these currents are quite unlikely to reach hazardous levels. The remote possibility that they might can be further reduced by controlled handling procedures in the clinical environment.

In ${ }^{13} \mathrm{C}$ - and ${ }^{1} \mathrm{H}$-NMR studies, broad band decoupling using higher power than the observation pulse will probably be necessary to suppress interfering peaks. Decoupling is potentially hazardous because it requires prolonged irradiation of the sample with radiofrequency energy, which at high power can cause heating of tissue. At present, it is possible to decouple anesthetized animals with no apparent adverse effects, and there is one report showing a decoupled spectrum of a human arm (36). Further careful studies with animals and the development of backup safety devices will be necessary before decoupling can be routinely used in humans.

\section{Future horizons}

Ultimately, it should be possible to combine NMR imaging and spectroscopic techniques so as to detect the distribution of metabolites within a particular organ. Such a capability would be invaluable for monitoring whole organ metabolism, the location and extent of tissue injury, and the effects of various treatment modalities. Such studies will be still more useful if they can be combined with NMR techniques for measuring blood flow (37). Multinuclear studies, e.g., ' $\mathrm{H}$ and ${ }^{13} \mathrm{C}$ or ${ }^{31} \mathrm{P}$ and ${ }^{13} \mathrm{C}$, using double- or even triple-tuned probes are feasible $(38,39)$, and they will increase the amount of information obtainable from NMR studies. ${ }^{19}$ F-NMR spectroscopy in biological systems is still relatively unexplored; it may allow pharmacologic study of fluorinated drugs by noninvasive detection of their distribution and metabolism (40). In vivo enzymologic studies of kinetics with magnetization transfer techniques (12) may be possible, as may studies of cellular compartmentation of metabolites using spin echo techniques (41).

In the last ten years, there has been an explosive growth in the use of NMR spectroscopy for the study of biological systems. With the development of NMR instruments suitable for human use, we are on the threshold of a new era in medical diagnosis and investigation of normal human metabolism.

\section{Acknowledgments}

The authors would like to thank Kevin Behar and Ognen Petroff for providing the spectrum for the figure, Tom Jue and Takashi Ogino for many helpful discussions, Leslie Vogel for the artwork, and Deborah Martone for typing the manuscript.

The work of the authors was supported by grants from the National Institutes of Health (AM-27121 and GM-30287), the National Science Foundation (PCM-8021715), and the Esther A. and Joseph Klingenstein Fund.

\section{References}

1. Ackerman, J. J. H., T. H. Grove, G. G. Wong, D. G. Gadian, and G. K. Radda. 1980. Nature (Lond.). 283:167-270.

2. Aue, W. P., S. Muller, T. A. Cross, and J. Seelig. 1984. J. Magn. Reson. 56:350-354.

3. Brown, T. R., B. M. Kincaid, and K. Ugurbil. 1982. Proc. Natl. Acad. Sci. USA. 79:3523-3526.

4. Maudsley, A. A., S. K. Hilal, W. H. Perman, and H. E. Simon.

1983. J. Magn. Reson. 51:147-152.

5. Bottomley, P. A. 1982. J. Magn. Reson. 50:335-338.

6. Scott, K. N., H. R. Brooker, J. R. Fitzsimmons, H. F. Bennett, and R. C. Mick. 1982. J. Magn. Reson. 50:339-344.

7. Haase, A., C. Malloy, and R. K. Radda. 1983. J. Magn. Reson. 55:164-169.

8. Bendel, P., C. M. Lai, and P. C. Lauterbur. 1980. J. Magn. Reson. 38:343-356.

9. Budinger, T. 1981. J. Comput. Assist. Tomogr. 5:800-811.

10. Moon, R. B., and J. H. Richards. 1973. J. Biol. Chem. 248:7276-7278.

11. Edwards, R. H. T., M. J. Dawson, D. R. Wilkie, R. E. Gordon, and D. Shaw. 1982. Lancet. I:725-731.

12. Shoubridge, E. A., R. W. Briggs, and G. K. Radda. 1982. FEBS Lett. 140:288-292.

13. Ugurbil, K., T. R. Brown, J. A. den Hollander, P. Glynn, and R. G. Shulman. 1978. Proc. Natl. Acad. Sci. USA. 75:3742-3746.

14. den Hollander, J. A., T. R. Brown, K. Ugurbil, and R. G. Shulman. 1979. Proc. Natl. Acad. Sci. USA. 76:6096-6100.

15. Neurohr, K. J., E. J. Barrett, and R. G. Shulman. 1983. Proc. Natl. Acad. Sci. USA. 80:1603-1607.

16. Behar, K. L., O. A. C. Petroff, J. R. Alger, J. W. Prichard, and R. G. Shulman. 1984. Magn. Reson. Med. 1:103. (Abstr.)

17. Cohen, S. M., S. Ogawa, and R. G. Shulman. 1979. Proc. Natl. Acad. Sci. USA. 76:1603-1607.

18. Cohen, S. M., P. Glynn, and R. G. Shulman. 1981. Proc. Natl. Acad. Sci. USA. 78:60-64.

19. Cohen, S. M., R. G. Shulman, and A. McLaughlin. 1979. Proc. Natl. Acad. Sci. USA. 76:4808-4812.

20. Cohen, S. M., R. Rognstad, R. G. Shulman, and J. Katz. 1981. J. Biol. Chem. 256:3428-3432.

21. Alger, J. R., L. O. Sillerud, K. L. Behar, R. J. Gillies, R. G. Shulman, R. E. Gordon, D. Shaw, and P. E. Hanley. 1981. Science (Wash. DC). 214:660-662.

22. Newman, R. J., P. J. Bore, L. Chan, D. G. Gadian, P. Styles, D. Taylor, and G. K. Radda. 1982. Br. Med. J. 284:1072-1074.

23. Behar, K. L., J. A. den Hollander, M. E. Stromski, T. Ogino, R. G. Shulman, O. A. C. Petroff, and J. W. Prichard. 1983. Proc. Natl. Acad. Sci. USA. 80:4945-4948.

24. Behar, K. L., D. L. Rothman, R. G. Shulman, O. A. C. Petroff, and R. G. Shulman. 1983. Proc. Natl. Acad. Sci. USA. 80:2748-2751.

25. Behar, K. L., O. A. C. Petroff, J. A. den Hollander, M. Stromski, J. W. Prichard and R. G. Shulman. 1983. Soc. Neurosci. Symp. 9(pt. 2):974. (Abstr.)

26. Ross, B. D., G. K. Radda, D. G. Gadian, G. Rocker, M. Esiri, and J. Falconer-Smith. 1981. N. Engl. J. Med. 304:1338-1342.

27. Radda, G. K., P. J. Bore, D. G. Gadian, B. D. Ross, P. Styles, D. J. Taylor, and J. Morgan-Hughes. 1982. Nature (Lond.). 295:608609. 
28. Cady, E. B., M. J. Dawson, P. L. Hope, P. S. Tofts, A. M. de L. Costello, D. T. Delpy, E. O. R. Reynolds, and D. R. Wilkie. 1983. Lancet. I:1059-1062.

29. Griffiths, J. R., E. Cady, R. H. T. Edwards, V. R. McCready, D. R. Wilkie, and E. Wiltshaw. 1983. Lancet. I:1435-36.

30. Stevens, A. N., R. A. Iles, P. G. Morris, and J. R. Griffiths. 1982. FEBS Lett. 150:489-493.

31. Alger, J. R., K. L. Behar, D. L. Rothman, and R. G. Shulman. 1984. J. Magn. Reson. 56:334-337.

32. Canioni, P., J. R. Alger, and R. G. Shulman. 1983. Biochemistry. 22:4974-4980.

33. Rothman, D. L., K. L. Behar, H. P. Hetherington, and R. G. Shulman. 1984. Soc. Magn. Reson. Med. In press.

34. Rothman, D. L., K. L. Behar, H. P. Hetherington, J. A. den
Hollander, M. R. Bendall, and R. G. Shulman. 1984. Magn. Reson. Med. In press.

35. Beischer, D. E. 1962. Astronautics. 7:24-25.

36. Gordon, R. E., P. E. Hanley, and D. Shaw. 1982. Prog. Nucl. Magn. Reson. Spectrosc. 15:1-47.

37. Morse, O. C., and J. R. Singer. 1970. Science (Wash. DC). 170:440-441.

38. den Hollander, J. A., K. L. Behar, and R. G. Shulman. 1984. J. Magn. Reson. 57:311-313.

39. Cohen, S. M. 1983. J. Biol. Chem. 258:14294-14308.

40. Wyrwicz, A. M., M. H. Pszenny, J. C. Scholfield, P. C. Tillman, R. E. Gordon, and P. A. Martin. 1983. Science (Wash. DC). 222:428430.

41. Brindle, K. M., F. F. Brown, I. D. Campbell, C. Grathwohl, and P. W. Kuchel. 1979. Biochem. J. 180:37-44. 